\title{
Potencial hidromineral dos aquíferos do estado de São Paulo
}

\author{
Lia Nogueira GARPELLI \& Didier GASTMANS
}

Laboratório de Recursos Hídricos e Isótopos Ambientais, Centro de Estudo Ambientais, Universidade Estadual Paulista "Júlio de Mesquita Filho". Av. 24 A, 1515, Bela Vista, CEP 13.506-900, Rio Claro, SP, Brasil (lia.garpelli@unesp.br, didier.gastmans@unesp.br).

\begin{abstract}
Resumo. 0 estado de São Paulo lidera a produção brasileira de água mineral. Devido ao seu complexo arcabouço hidrogeológico, composto por vários aquíferos sedimentares e fraturados, as águas minerais apresentam diferentes características hidrominerais. Assim, o principal objetivo foi avaliar o potencial hidromineral dos aquíferos paulistas, fundamentado pelo Código de Águas Minerais (CAM) e legislações internacionais correlatas, a partir de uma base de dados pública de laudos analíticos de 276 poços de águas subterrâneas da Companhia Ambiental do Estado de São Paulo (CETESB). Baseado nas concentrações de Sólidos Totais Dissolvidos (STD), observou-se que as águas subterrâneas possuem grau variável de mineralização, sendo as águas mais mineralizadas associadas aos sistemas aquíferos Serra Geral (SASG), Tubarão (SAT) e Bauru (SAB). Entretanto, apenas 8,7\% das águas se enquadrariam como mínimo e baixo teores de STD pela legislação dos Estados Unidos. Similarmente, apenas duas amostras seriam enquadradas como muito pouco mineralizadas e $81,5 \%$ em pouco mineralizadas pela legislação europeia. Pelas classificações dispostas pelo CAM, as amostras foram caracterizadas pela temperatura, predominantemente, como frias e hipotermais, e uma pequena parcela como mesotermais e hipotermais. As áquas mais frias prevaleceram no aquífero Cristalino (SAC), enquanto as águas de maiores temperaturas ocorreram no Guarani (SAG). Todo os aquíferos possuíram potencial hidromineral pela classificação química, pela concentração de flúor, seguido em ordem decrescente, pelo lítio no SAC, o vanádio no SASG, a alcalina bicarbonato no SAT, sulfato e selênio de forma mista no SAT e SASG.
\end{abstract}

Palavras-chave. Águas subterrâneas, Código de Águas Minerais, Hidroquímica, Estado de São Paulo

\begin{abstract}
Hydromineral POTENTIAL of SÃo PAULo StATE aquifERS. The state of São Paulo leads the Brazilian production of bottled mineral water. Due to its complex hydrogeological framework, there is a variety of sedimentary and fractured aquifers, which can contribute to occurrences of waters with different hydromineral characteristics. Thus, the main objective was to evaluate the hydromineral potential of the São Paulo aquifers, according to the Mineral Water Code (CAM) and related international legislation, based on a public database of analytical reports from 276 underground water wells of Environmental Company of the State of São Paulo (CETESB). When analyzed on the concentrations of Total Dissolved Solids (TDS), it was observed that groundwater had a varying degree of mineralization, with the most mineralized waters associated with Serra Geral (SASG), Tubarão (SAT) and Bauru (SAB) aquifer systems. However, only $8.7 \%$ of the waters would be qualify as minimum to low levels of TDS, under United States legislation. Similarly, only two samples would be classified as very low mineralized and, $81.5 \%$ as low mineralized, by European legislation. By the classifications provided by CAM, the samples were characterized by temperature, predominantly, as cold and hypothermal, and a small portion as mesothermal and hypothermal. The colder waters prevailed in Cristalino aquifer (SAC), while the higher temperatures occurred in Guarani (SAG). All aquifers had hydromineral potential by chemical classification, due to fluoride concentration, followed in decreasing order, by lithium in SAC, vanadium in SASG, alkaline bicarbonate in SAT, sulfate and selenium as a mixed water in SAT and SASG.
\end{abstract}

Keywords. Groundwater, Brazilian Mineral Water Code, Hydrochemical, State of São Paulo. 


\section{Introdução}

A água é um recurso essencial à sobrevivência do homem, importante para o desenvolvimento das sociedades e nações. Em função de suas características físico-químicas e disponibilidade, é utilizada em diversas atividades humanas, como: consumo das populações, atividades agrícolas, na geração de energia elétrica, como insumo na atividade industrial e também na recreação, além de ter um papel ecossistêmico, pouco valorado nos dias atuais, (Porowski et al., 2019).

As águas subterrâneas, armazenadas nos poros e fissuras das rochas, possuem características singulares, e vêm desde há muito tempo sendo aproveitadas para consumo e fins terapêuticos, devido às suas características físico-químicas, derivadas da capacidade solvente da água, que em contato com as rochas dos aquíferos vão gradualmente adquirindo características físico-químicas e concentrações em sais que thes confere composição química e propriedades terapêuticas únicas, e as caracterizando como águas minerais (Selinus, 2005; Shvartsev, 2008).

Entretanto, a definição de água mineral não é consensual, variando em função da sua utilização ou campo de aplicação, se em hidrogeologia, balneologia ou na indústria de alimentos (água engarrafada). O termo é tradicionalmente utilizado para definir águas subterrâneas com propriedades curativas, e que possuam concentrações superiores à média em elementos dissolvidos, gases e/ou com temperaturas elevadas. Atualmente, o termo vem sendo globalmente utilizado para separar as águas naturais das subterrâneas, definindo as águas minerais como aquelas com concentrações superiores a $1 \mathrm{~g} \mathrm{~L}^{-1}$ de sólidos totais dissolvidos, ou conteúdos significativos elementos químicos com propriedades medicinais (Porowski, 2018).

As águas subterrâneas, quando caracterizadas como minerais, são alocadas no contexto de jazidas minerais e regidas por leis específicas, para assegurar a qualidade do produto e a saúde do consumidor. Dependendo do país da produção, as regulamentações referentes às concentrações de elementos para seu enquadramento como água mineral variam. No Brasil, os critérios para determinação das águas minerais são estabelecidos pelo Decreto Lei 7.841, 08/08/1945, o Código de Água Mineral (CAM), Brasil (1945), e pela Portaria do Departamento Nacional de Produção Mineral (DNPM), atual Agência Nacional de Mineração (ANM) no 540 de 18 de dezembro de 2014, (Brasil, 2014).

A utilização das águas minerais mudou com o tempo, passando de fins curativos e balneários, importante até o final do século 19, para um importante setor da economia: a indústria de água mineral engarrafada. Em 2016, o consumo das águas engarrafas no mundo atingiu 346 bilhões de litros, sendo o mercado brasileiro o quinto do mundo, à frente de países como Índia, Tailândia, Alemanha e Itália (Rodwann Jr., 2018). O estado de São Paulo lidera a produção brasileira, engarrafando cerca de $22 \%$ de toda a água mineral consumida no país. Embora o consumo interno seja alto, superior à 20 bilhões de litros, a exportação de águas minerais ainda é incipiente, não ultrapassando 716 mil litros, apesar das reservas existentes (DNPM, 2017).

Em função do arcabouço hidrogeológico do estado de São Paulo, as águas subterrâneas, além de serem aproveitadas como águas minerais, abastecem, total ou parcialmente, cerca de $80 \%$ dos municípios do estado (CETESB, 2010). Desse modo é notável a importância das águas subterrâneas no Estado, seja como recurso mineral, seja como recurso hídrico. A caracterização e evolução hidroquímica das águas subterrâneas de diferentes aquíferos do estado de São Paulo foi extensivamente estudada por diversos autores (Vidal \& Chang, 2002; Barison \& Chang, 2003; Ezaki et al., 2014; Chang et al., 2016; Quaggio et al., 2018; Roque et al., 2018; e apenas o SAG teve seu potencial hidromineral no estado avaliado (Gastmans et al., 2012).

O presente trabalho pretende preencher essa lacuna do conhecimento, apresentando uma avaliação do potencial hidromineral dos aquíferos do estado de São Paulo, baseado em dados públicos, e responder algumas questões científicas relevantes em relação a mineralização das águas subterrâneas do estado de São Paulo, 
como: bases de dados hidroquímicos públicas constituem ferramenta importante na avaliação do potencial mineral de aquíferos?; quais são os elementos presentes nas águas subterrâneas do estado de São Paulo que conferem características minerais a suas águas?; considerando o complexo arcabouço hidrogeológico do estado de São Paulo, é possível estabelecer um zoneamento hidromineral?; as unidades aquíferas presentes no estado armazenam águas minerais com distintas classificações?.

Desse modo, o objetivo do trabalho é classificar as águas subterrâneas do estado de São Paulo, segundo o código vigente de águas minerais (CAM), Brasil (1945), avaliando seu potencial mineral, por meio dos principais elementos químicos dissolvidos e/ ou propriedades químicas que as caracterizam, possibilitando a confecção de um produto cartográfico que possa orientar a prospecção das águas minerais no estado, além de avaliar se essas águas seriam classificadas como minerais em regulamentações internacionais consagradas.

\section{Arcabouço hidrogeológico do estado de São} Paulo

Localizada na região Sudeste do Brasil, o estado de São Paulo faz divisas com os estados do Rio de Janeiro, Paraná, Minas Gerais e Mato Grosso do Sul. Localizada entre os paralelos $20^{\circ}$ e $25^{\circ} \mathrm{S}$ e os meridianos $44^{\circ} 30^{\prime}$ e $53^{\circ} \mathrm{W}$. Concentra cerca de $22 \%$ da população brasileira, distribuídos em 645 municípios. Além disso, possui os principais centros financeiros, industriais e difusão de informação e pesquisa, com o maior PIB e o segundo maior IDH do país IBGE (2017).

No estado há uma certa diversidade climática, em consequência da variedade de relevos, posição geográficaediferentes influências de massas de ar. Através da classificação de Köppen, é dividido em tropical (A) e subtropical (C), com variações quanto ao regime de chuvas e temperatura. Há uma predominância dos climas Tropical quente com inverno seco (Aw) no norte e nordeste e, Subtropical quente e úmido (Cfa), no centro-leste do estado. Outras classificações ocorrem, contudo, em menor escala. Como tropical sem estação seca (Af), no litoral sul; e com chuvas excessivas e inverno seco (Am), no litoral norte, Vale do Ribeira, e região próxima ao norte do estado do Paraná. Dos subtropicais, o temperado sem estação seca (Cfb) ocorre na faixa de latitude da cidade de São Paulo e porções altas da Mata Atlântica; as demais subtropicais de inverno seco ocorrem em regiões próximas do sul de Minas Gerais e Rio de Janeiro (Cwa e Cwb) de temperatura quente e temperado, respectivamente (Rolim et al., 2007).

O embasamento cristalino e parte da Bacia do Paraná delimitam os dois principais compartimentos hidrogeológicos do território paulista (Fig. 1), e com base na litologia dos terrenos e suas propriedades hidráulicas, o estado possui dois tipos principais de aquíferos: os fraturados, permeáveis por fissuramento das rochas, e os sedimentares que são permeáveis por porosidade granular, contando ainda com um aquiclude de caráter passivo (aquiclude Passa Dois) (Campos, 1993; DAEE et al., 2005; Iritani \& Ezaki, 2009; CETESB, 2010, 2016).

Os aquíferos fissurais do estado de São Paulo são representados pelos sistemas aquíferos Cristalino (SAC) e Serra Geral (SASG), localizados respectivamente na porção leste do estado e na porção central (Fig. 1). Os aquíferos sedimentares estão divididos entre aqueles associados as bacias sedimentares terciárias: sistemas aquíferos São Paulo (SASP) e Taubaté (SATA), e os associados à Bacia Sedimentar do Paraná, que ocupa toda a porção oeste do estado, representados pelos sistemas aquíferos Tubarão (SAT), Guarani (SAG) e Bauru (SAB). Sucintamente, as características gerais dos sistemas aquíferos do trabalho estão descritas no quadro 1, com as litologias que os constituem, suas propriedades hidrogeológicas e hidráulicas, assim como as principais características físicoquímicas de suas águas.

\section{Material e métodos}

A avaliação realizada no presente estudo tem como base de dados os laudos analíticos de amostras de águas subterrâneas coletadas no segundo semestre 


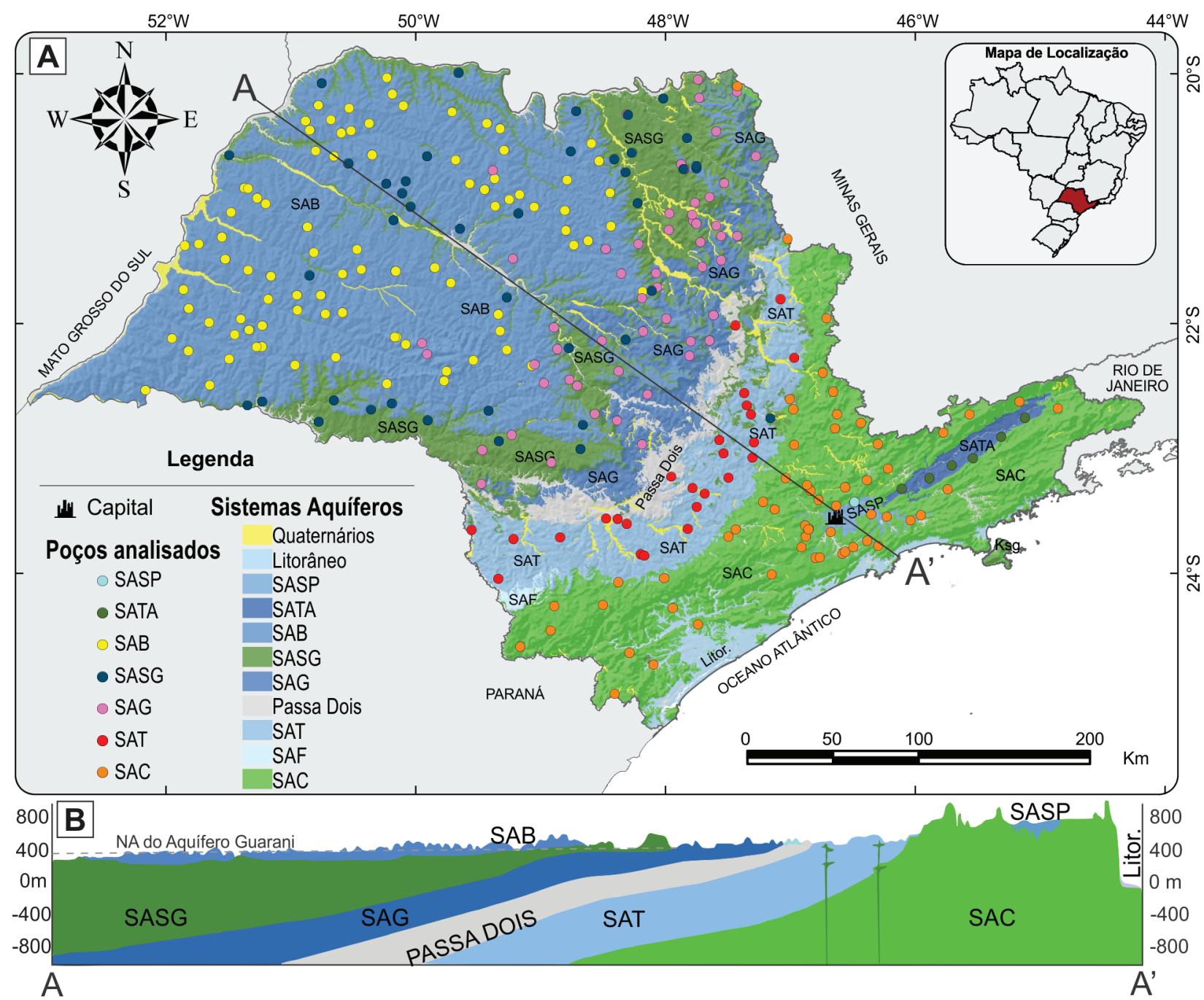

Figura 1. Mapa hidrogeológico do estado de São Paulo. A) Distribuição dos poços analisados no trabalho. B) Perfil esquemático A-A' da disposição dos aquíferos. (Modificado de DAEE/UNESP, 1980; DAEE et al., 2005). Segundo convenção internacional, os aquíferos granulares têm cor azul e os aquíferos fraturados têm cor verde. As diferentes tonalidades correspondem à variação de produtividade no âmbito dos aquíferos: quanto mais escuras, maior produtividade. Abreviação dos Sistemas Aquíferos: SASP. São Paulo; SATA: Taubaté; SAB: Bauru; SASG: Serra Geral; SAG: Guarani; SAT: Tubarão; SAF: Furnas; SAC: Cristalino.

Figure 1. Hydrological map of State of São Paulo. A) Distribution of analyzed wells in this work. B) A-A' Schematic profile of the aquifers disposition (Modified from DAEE/UNESP, 1980; DAEE et al., 2005). According to international convention, the sedimentary aquifers have blue color and the fractured aquifers have green ones. The different nuances correspond the producibility variation: when darker, greater production. Abbreviation of the Aquifer Systems: SASP. São Paulo; SATA: Taubaté; SAB: Bauru; SASG: Serra Geral; SAG: Guarani; SAT: Tubarão; SAF: Furnas; SAC: Cristalino.

de 2015 pela CETESB em sua Rede de Monitoramento de Qualidade (disponível em https://cetesb.sp.gov.br/wp-content/ uploads/2017/09/Apendice-A-ResultadosAnal\%C3\%ADticos-2013-2015.zip).

Foram analisados resultados analíticos de 276 poços amostrados em 250 cidades no estado (Fig. 1), dos quais 88 análises são representativas do SAB; 53 do SAG, 60 do SAC, 42 do SASG, 25 do SAT, 6 do SATA e 2 do SASP.

A classificação das amostras foi realizada segundo os critérios do Código de Águas
Minerais de 1945, CAM (Brasil, 1945), que adota como principais critérios as características conservativas (constituintes químicos) da água e aquelas não conservativas ou inerentes às fontes, como presença de gases e temperatura. Em seu Artigo 35, o CAM ainda define que as águas minerais serão classificadas pela ANM pelo elemento predominante na água, podendo ter classificação mista as que acusarem mais de um elemento digno de nota, ou com substâncias raras dignas de nota. Esses elementos são apresentados no quadro 2. 
Quadro 1. Síntese das características gerais dos principais sistemas aquíferos do estado de São Paulo (Compilado de Campos, 1993; CETESB, 1998, 2016; CERH, 1999; e DAEE et al., 2005). Abreviação: SA: Sistema Aquífero; Q/s: Vazão Específica; STD: Sólidos Totais Dissolvidos; CH: Classificação Hidroquímica; Bic.: Bicarbonatada; e dos sistemas aquíferos SASP. São Paulo; SATA: Taubaté: SAB: Bauru; SASG: Serra Geral: SAG: Guarani: SAT: Tubarão: SAC: Cristalino. Chart 1. General characteristics synthesis of the principal aquifer systems of State of São Paulo (Compiled from Campos, 1993; CETESB, 1998, 2016; CERH, 1999; e DAEE et al., 2005). Abbreviation SA: Aquifer System; Q/s: Specific Discharge; STD (as Portuguese abbreviation for TDS): Total Dissolved Solids; CH: Hydrochemical Classification; Bic: Bicarbonate; and the aquifer systems, SASP. São Paulo; SATA: Taubaté: SAB: Bauru; SASG: Serra Geral: SAG: Guarani: SAT: Tubarão: SAC: Cristalino

\begin{tabular}{|c|c|c|c|}
\hline $\mathbf{S A}$ & Litologia & $\begin{array}{l}\text { Características } \\
\text { Hidrogeológicas }\end{array}$ & Demais Propriedades \\
\hline$\sum_{\sqrt[n]{2}}^{\frac{1}{4}}$ & $\begin{array}{l}\text { Arenitos de granulação variável, } \\
\text { argilas e cascalhos }\end{array}$ & $\begin{array}{c}\text { Extensão limitada, } \\
\text { granular, livre, } \\
\text { descontínuo, heterogêneo, } \\
\text { anisotrópico }\end{array}$ & $\begin{array}{l}\text { Q/s: } 0,5-1,0 \mathrm{~m}^{3} \mathrm{~h}^{-1} \mathrm{~m}^{-1} ; \mathrm{pH}: 6,4 \mathrm{a} \\
7,8 \\
\text { STD: } 82-154 \mathrm{mg} \mathrm{L}^{-1}\end{array}$ \\
\hline 造 & $\begin{array}{c}\text { Arenitos, lentes de folhelhos, } \\
\text { argilitos localmente } \\
\text { pirobetuminosos com intercalações } \\
\text { subordinadas de brechas } \\
\text { sedimentares e conglomerados }\end{array}$ & $\begin{array}{c}\text { Extensão limitada, } \\
\text { granular, livre a semi- } \\
\text { confinado, descontínuo, } \\
\text { heterogêneo, anisotrópico }\end{array}$ & $\begin{array}{l}\text { Q/s: } 0,2-14,0 \mathrm{~m}^{3} \mathrm{~h}^{-1} \mathrm{~m}^{-1} ; \mathrm{pH}: 6,0- \\
7,7 ; \text { STD: } 50-160 \mathrm{mg} \mathrm{L}^{-1} \text {; } \mathrm{CH}: \\
\text { Bic. cálcica }(20 \%) \text {, Bic. sódica } \\
(42 \%)\end{array}$ \\
\hline$\sum_{\infty}^{\infty}$ & $\begin{array}{c}\text { Predominância de arenitos de } \\
\text { granulação fina a grossa, } \\
\text { localmente mal selecionados, e por } \\
\text { vezes com intercalações de lentes } \\
\text { de siltito, argilitos }\end{array}$ & $\begin{array}{l}\text { Extensão regional, granular } \\
\text { livre a semi-confinado, } \\
\text { descontínuo, heterogêneo, } \\
\text { anisotrópico. Aquífero } \\
\text { freático com recarga } \\
\end{array}$ & $\begin{array}{l}\text { Q/s: } 0,5-2 \mathrm{~m}^{3} \mathrm{~h}^{-1} \mathrm{~m}^{-1} ; \mathrm{pH}: 4,59- \\
\text { 9,64; STD: }<300 \mathrm{mg} \mathrm{L}^{-1} \\
\text { CH: Bic. cálcica }(58 \%), \text { Bic. } \\
\text { cálcico-magnesianas }(11 \%)\end{array}$ \\
\hline ए & $\begin{array}{c}\text { Rochas vulcânicas toleíticas em } \\
\text { derrames basálticos, com } \\
\text { intercalações de arenitos } \\
\text { intertrapeanos, finos a médios }\end{array}$ & $\begin{array}{c}\text { Extensão limitada, fissural, } \\
\text { caráter eventual, livre a } \\
\text { semi-confinado, } \\
\text { descontínuo, heterogêneo, } \\
\text { anisotrópico }\end{array}$ & $\begin{array}{l}\mathrm{Q} / \mathrm{s}: 0,01-0,62 \mathrm{~m}^{3} \mathrm{~h}^{-1} \mathrm{~m}^{-1} ; \mathrm{pH}: \\
\text { 6,0-7,0; STD: }<200 \mathrm{mg} \mathrm{L}^{-1} \\
\mathrm{CH}: \text { Bic. cálcicas, } \\
\text { secundariamente magnesianas }\end{array}$ \\
\hline 幽 & $\begin{array}{c}\text { Arenitos avermelhados finos a } \\
\text { médios, com intercalações areno- } \\
\text { conglomerática, níveis de porções } \\
\text { mais finas, com arenitos argilosos, } \\
\text { siltitos e folhelhos }\end{array}$ & $\begin{array}{l}\text { Extensão regional, granular } \\
\text { livre a confinado, } \\
\text { homogêneo, contínuo, } \\
\text { isotrópico }\end{array}$ & $\begin{array}{l}\mathrm{Q} / \mathrm{s}: 0,01-26,0 \mathrm{~m}^{3} \mathrm{~h}^{-1} \mathrm{~m}^{-1} ; \mathrm{pH}: \\
\text { 6,0-7,0 ; STD: }<200 \mathrm{mg} \mathrm{L}^{-1} ; \\
\text { CH: Bic. cálcicas, } \\
\text { secundariamente magnesianas }\end{array}$ \\
\hline 点 & $\begin{array}{l}\text { Grande diversidade litológica, com } \\
\text { arenitos médios a grossos } \\
\text { feldspáticos, arenitos finos, } \\
\text { conglomerados, siltitos, folhelhos } \\
\text { rítmicos, diamictitos, calcários, } \\
\text { tilitos e raras camadas de carvão }\end{array}$ & $\begin{array}{c}\text { Extensão regional, granular } \\
\text { livre a semi-confinado, } \\
\text { heterogêneo, descontínuo, } \\
\text { anisotrópico (localmente } \\
\text { fissurado, descontínuo e } \\
\text { isotrópico) }\end{array}$ & $\begin{array}{l}\text { Q/s: } 0,002-4,67 \mathrm{~m}^{3} \mathrm{~h}^{-1} \mathrm{~m}^{-1} ; \mathrm{pH}: \\
4,5-10 ; \text { STD: } 50-629 \mathrm{mg} \mathrm{L}^{-1} ; \\
\text { CH: Bic. sódica e } \\
\text { secundariamente Bic. cálcica } \\
\text { ou mistas }\end{array}$ \\
\hline$\underset{\sim}{u}$ & $\begin{array}{l}\text { Rochas pré-cambrianas ígneas e } \\
\text { metamórficas como granitos, } \\
\text { granitoides, gnaisses, migmatitos, } \\
\text { filitos, xistos, quartzitos e } \\
\text { metassedimentos }\end{array}$ & $\begin{array}{c}\text { Extensão regional, fissural, } \\
\text { caráter eventual, livre a } \\
\text { semi-confinado, } \\
\text { heterogêneo, descontínuo, } \\
\text { anisotrópico }\end{array}$ & $\begin{array}{l}\text { Q/s: } 0,04-0,18 \mathrm{~m}^{3} \mathrm{~h}^{-1} \mathrm{~m}^{-1} ; \mathrm{pH}: \\
\text { 5,0-9,4; } \mathrm{STD} 50-334 \mathrm{mg} \mathrm{L}^{-1} \text {; } \\
\text { CH: Bic. cálcicas, } \\
\text { secundariamente sódicas }\end{array}$ \\
\hline
\end{tabular}


Quadro 2. Concentrações de alguns elementos utilizados nesse trabalho para classificação das águas minerais, segundo o CAM. Não consta a classificação das nitratadas, sulfurosas, radíferas, radioativas, e oligominerais (modificado do Brasil, 1945, 2014).

Chart 2. Concentration of some elements used in this work to classify mineral waters, according to CAM. It does not contain the classification of nitrated, sulfurous, with radium, radioactive, and oligomineral (modified from Brasil, 1945, 2014).

\begin{tabular}{|c|c|c|c|}
\hline \multicolumn{2}{|c|}{$\begin{array}{c}\text { Classificação das águas } \\
\text { minerais }\end{array}$} & \multirow{2}{*}{\begin{tabular}{|c|}
$\begin{array}{c}\text { Concentração mínima do íon } \\
\text { predominante }\left(\mathrm{mg} \mathrm{L}^{-1}\right)\end{array}$ \\
$0,02 \mathrm{mg} \mathrm{L}^{-1}($ de $\mathrm{F})$ \\
\end{tabular}} & \multirow{2}{*}{$\begin{array}{l}\text { Principal composto } \\
\text { Segundo CAM e } \\
\text { Portaria } n^{\circ} 540 \\
\text { F }\end{array}$} \\
\hline \multirow{4}{*}{$\begin{array}{l}\text { Dignos } \\
\text { de nota }\end{array}$} & Fluoreto & & \\
\hline & Lítio & $0,01 \mathrm{mg} \mathrm{L}^{-1}$ (de Li) & $\mathrm{Li}$ \\
\hline & Vanádio & $0,03 \mathrm{mg} \mathrm{L}^{-1}(\mathrm{de} \mathrm{V})$ & $\mathrm{V}$ \\
\hline & Selênio & $0,006 \mathrm{mg} \mathrm{L}^{-1}(\mathrm{de} \mathrm{Se})$ & $\mathrm{Se}$ \\
\hline \multicolumn{2}{|c|}{ Alcalina bicarbonatada } & $\begin{array}{l}145 \mathrm{mg} \mathrm{L}^{-1}\left(\mathrm{de} \mathrm{HCO}_{3}\right) \text { e } 55 \mathrm{mg} \mathrm{L}^{-1} \text { (de } \\
\mathrm{Na} \text { ) }\end{array}$ & $0,2 \mathrm{~g} \mathrm{~L}^{-1}$ de $\mathrm{NaHCO}_{3}$ \\
\hline \multirow{2}{*}{$\begin{array}{l}\text { Alcalina } \\
\text { terrrosa }\end{array}$} & Cálcicas & $\begin{array}{l}119 \mathrm{mg} \mathrm{L}^{-1} \text { (de Ca) e } 361 \mathrm{mg} \mathrm{L}^{-1} \mathrm{mg} \mathrm{L}^{-1} \\
\left(\text { de } \mathrm{HCO}_{3}\right)\end{array}$ & $\begin{array}{l}0,48 \mathrm{~g} \mathrm{~L}^{-1} \text { de } \mathrm{Ca} \text { em } \\
\mathrm{Ca}\left(\mathrm{HCO}_{3}\right)_{2}\end{array}$ \\
\hline & Magnesianas & $\begin{array}{l}8,6 \mathrm{mg} \mathrm{L}^{-1}(\mathrm{de} \mathrm{Mg}) \text { e } 21,4 \mathrm{mg} \mathrm{L}^{-1}(\mathrm{de} \\
\left.\mathrm{CO}_{3}\right)\end{array}$ & $0,03 \mathrm{~g} \mathrm{~L}^{-1}$ de $\mathrm{MgCO}_{3}$ \\
\hline \multicolumn{2}{|c|}{ Sulfatada } & $\begin{array}{l}100 \mathrm{mg} \mathrm{L}^{-1}\left(\mathrm{de} \mathrm{SO}_{4}\right) ; 23,94 \mathrm{mg} \mathrm{L}^{-1}(\mathrm{de} \\
\mathrm{Na}) ; 25,32 \mathrm{mg} \mathrm{L}^{-1}(\mathrm{de} \mathrm{Mg}) ; \text { ou } 40,64 \mathrm{mg} \\
\mathrm{L}^{-1} \text { (de K) }\end{array}$ & $\begin{array}{l}0,1 \mathrm{~g} \mathrm{~L}^{-1} \text { de } \mathrm{SO}_{4} \mathrm{cl} \\
\mathrm{Na}, \mathrm{Mg} \text { ou } \mathrm{K}\end{array}$ \\
\hline \multicolumn{2}{|c|}{ Cloretadas } & $\begin{array}{l}\text { 196,5 } \mathrm{mg} \mathrm{L}^{-1} \text { (de } \mathrm{Na} \text { ) e } 303,5 \mathrm{mg} \mathrm{L}^{-1} \text { (de } \\
\mathrm{Cl})\end{array}$ & $0,5 \mathrm{~g} \mathrm{~L}^{-1} \mathrm{NaCl}$ \\
\hline \multicolumn{2}{|c|}{ Ferruginosa } & $500 \mathrm{mg} \mathrm{L}^{-1}(\mathrm{de} \mathrm{Fe})$ & $\mathrm{Fe}$ \\
\hline
\end{tabular}

De acordo com o relatório da CETESB (2016), os resultados analíticos possuem limites de quantificação (LQ) diferentes para uma mesma substância, em decorrência das diferentes técnicas e métodos utilizados pelos diferentes laboratórios da CETESB, responsáveis pelas análises químicas. Desse modo, embora o LQ para fluoreto ainda seja alto $(0,1$ e 0,5 mg $\left.\mathrm{L}^{-1}\right)$ para determinação das águas como minerais $\left(0,02 \mathrm{mg} \mathrm{L}^{-1}\right)$, optou-se para exclusão dos valores abaixo do LQ. O estudo também não considerou as análises de qualidade e potabilidade.

A classificação das fontes quanto as temperaturas, seguem em: Fontes frias as que tiverem temperaturas menores que $25^{\circ} \mathrm{C}$; Hipotermais com temperaturas entre 25 a $33^{\circ} \mathrm{C}$; Mesotermais com 33 a $36{ }^{\circ} \mathrm{C}$; Isotermais, com temperaturas entre 36 a $38^{\circ} \mathrm{C}$, e; Hipertermais as fontes de água com temperatura superior à 38 ${ }^{\circ} \mathrm{C}$.

Como etapa final, a elaboração de mapas temáticos gerados pelo software ArcGis, versão 10.5, e apresentados em Sistema de Coordenadas Geográficas GCS SIRGAS 2000. Os arquivos que compuseram o banco de dados para realização dos mapas foram dados analíticos físico-químicos dos poços, shapefiles dos domínios hidrogeológicos, modificados de
DAEE/UNESP (1980) e DAEE et al. (2005), e relevo sombreado obtido a partir de dados de domínio público do U.S Geological Survey do Modelo Digital de Terreno, do Shuttle Radar Topography Mission (SRTM).

Uma avaliação hidroquímica e de qualidade dessas águas não é objetivo do presente estudo, e detalhes podem ser consultados diretamente no relatório de qualidade das águas subterrâneas no estado de São Paulo (CETESB, (2016).

\section{Resultados e discussões}

As águas subterrâneas do estado de SP são consideradas como águas que apresentam, de modo geral, baixas mineralizações, traduzidas por STD variando entre 50 e $680 \mathrm{mg} \mathrm{L}^{-1}$, e médias entre 114 a 189,6 $\mathrm{mg} \mathrm{L}^{-1}$ de acordo com o aquífero explorado (Fig. 2A e B). Esses valores são similares aos medidos por diversos autores nos vários aquíferos presentes no estado (Szikszay \& Teissedre, 1981; Campos, 1993; Vidal \& Chang, 2002; Barison \& Chang, 2003; Bertolo et al., 2007; Gastmans et al., 2013; Ezaki et al., 2014; Chang et al., 2016; Bulia \& Enzweiler, 2018; Quaggio et al., 2018).

Embora não haja uma diretriz brasileira, a ANM, em um estudo diagnóstico das águas 
minerais brasileiras, (DNPM, 2015), propõe rotulá-las pelo teor de sais - entre muito baixo (<50 $\left.\mathrm{mg} \mathrm{L}^{-1}\right)$, baixo (50 a $100 \mathrm{mg} \mathrm{L}^{-1}$ ), médio (100 a $200 \mathrm{mg} \mathrm{L}^{-1}$ ) e alto (> $200 \mathrm{mg} \mathrm{L}^{-1}$ ). Desse modo, a variação da concentração de sais permite classificá-las em todas as categorias. Com grande representação das águas que apresentam concentrações médias de sais, destaca-se as águas subterrâneas do SAB e SAC (Fig. 2C). Algumas águas dos sistemas aquíferos apresentam valores de STD elevados, com 448 $\mathrm{mg} \mathrm{L}^{-1}$ no SAB, 494 e $586 \mathrm{mg} \mathrm{L}^{-1}$ no SAT e $680 \mathrm{mg}$ $L^{-1}$ no $S A S G$, que permitiram sua classificação como águas com altas concentrações de sais.

A concentração de STD é um importante parâmetro para classificar a mineralização das águas. A caracterização das águas tanto nos Estados Unidos, quanto na Comunidade Europeia é baseada, principalmente, pela concentração de STD, diferentemente do Brasil, que não leva em consideração esse valor (Parlamento Europeu, 2009; U.S. Federal Government, 2019). Essa ausência de valores, pode ser um empecilho no que tange à comercialização das águas internacionalmente. Comparativamente, as diretrizes dos três países estão resumidas no quadro 3. Essa observação, contudo, não desvaloriza a qualidade da água mineral brasileira. Segundo o relatório do Laboratório de Análises Minerais, do Serviço Geológico Brasileiro (LAMIN, 2015), o Brasil vem ganhando atenção de grandes grupos internacionais, na pesquisa de fontes com baixos valores de resíduo seco (ou STD).

Quando comparadas a classificações de outros países, apenas 24 amostras se enquadrariam como mineral, com concentração mínima de $250 \mathrm{mg} \mathrm{L}^{-1}$ de sais dissolvidos, pela legislação americana. Dessas, 22 amostras seriam classificadas como água mineral de baixo teor mineral (até $500 \mathrm{mg} \mathrm{L}^{-1}$ ) (Fig. 2D). De modo similar, de acordo com a legislação europeia, apenas 24 amostras seriam classificadas segundo sua concentração de sais, duas como muito pouco mineralizadas (concentrações de até $50 \mathrm{mg} \mathrm{L}^{-1}$ ), e 22 em oligominerais, ou pouco mineralizadas (concentrações de até $500 \mathrm{mg} \mathrm{L}^{-1}$ ) (Fig. 2E).

As águas subterrâneas apresentam temperaturas que variaram entre 18 e $44,8^{\circ} \mathrm{C}$, com a média, mediana e $3^{\circ}$ quartil, apresentando valores muitos próximos $(25,17$; 25,2; e 26,2 ${ }^{\circ} \mathrm{C}$, respectivamente). Os poços com maiores temperaturas pertencem ao SAG, com $40,9^{\circ} \mathrm{C}$ e $44,8^{\circ} \mathrm{C}$, em que a água mais quente foi amostrada em uma profundidade de captação entre 721 e $1292 \mathrm{~m}$. A temperatura mais baixa registrada foi no SAC, com $18^{\circ} \mathrm{C}$, em uma profundidade que não ultrapassa $43 \mathrm{~m}$, o que permite enquadrar as águas subterrâneas em quatro classes: hipotermais, frias, mesotermais e hipertermais (Fig. 3).

Mais da metade das amostras (53,6\%), especialmente no SAB, apresentaram temperaturas entre 25 a $33{ }^{\circ} \mathrm{C}$, classificadas como hipotermais, seguidas das águas frias com 44,9\% das amostras, predominantemente no SAC. As águas mais quentes estão no SAG, contudo, apresentam uma pequena porção do total, e são classificadas como mesotermal (uma amostra - 0,36\%), e hipertermais representando $0,75 \%$ do total.

Quantoà classificação química preconizada pelo CAM (Brasil, 1945), as águas subterrâneas do estado podem ser classificadas em seis tipos distintos: águas fluoretadas, litinadas, vanádicas, sulfatadas, alcalina-bicarbonatadas e seleniadas (Fig. 4).

Embora o LQ dos dados analíticos do fluoreto sejam altos $\left(0,1\right.$ e 0,5 $\mathrm{mg} \mathrm{L}^{-1}$ de $\left.\mathrm{F}^{-}\right)$, que permitiria enquadrar todas as amostras como fluoretadas - visto a baixa exigência do código de águas (concentração de $\mathrm{F}^{-}$acima de 0,02 mg $L^{-1}$ ) - optou-se pela retirada das amostras em que as concentrações medidas não atingiram os limites de quantificação do equipamento. Desse modo, 28,98\% das águas foram classificadas como fluoretadas, numa concentração média de $0,53 \mathrm{mg} \mathrm{L}^{-1}$. Todos os aquíferos entraram na classificação, com destaque para as amostras do SAC, que compôs a maioria dos pontos (com 34 poços), seguido do SAB na porção norte, e secundariamente, o SAT, SASG, SAG, SATA e SASP (Fig. 4). Apenas três amostras apresentaram concentrações do elemento acima do recomendado para consumo diário $\left(1,5 \mathrm{mg} \mathrm{L}^{-1}\right.$ de acordo com a Portaria de Consolidação n5 do Ministério da Saúde). 


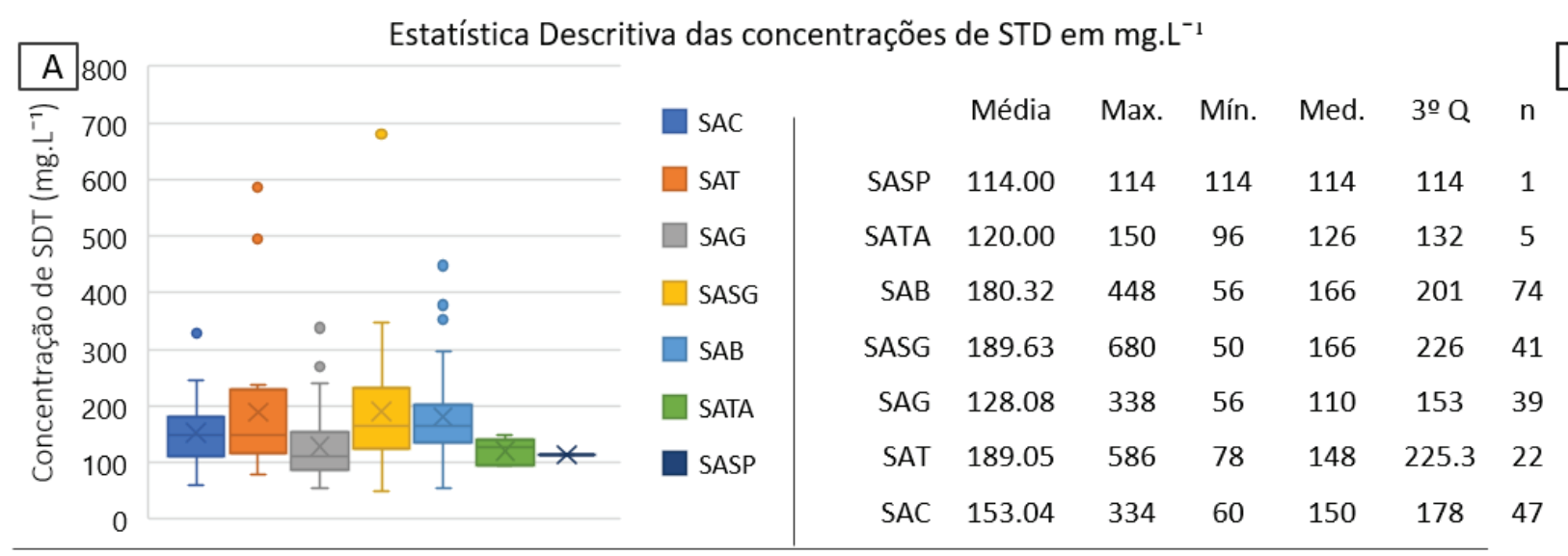

C Classificação das águas pelas concentrações de STD (mg. $\left.\mathrm{L}^{-1}\right)$, de acordo com diretrizes

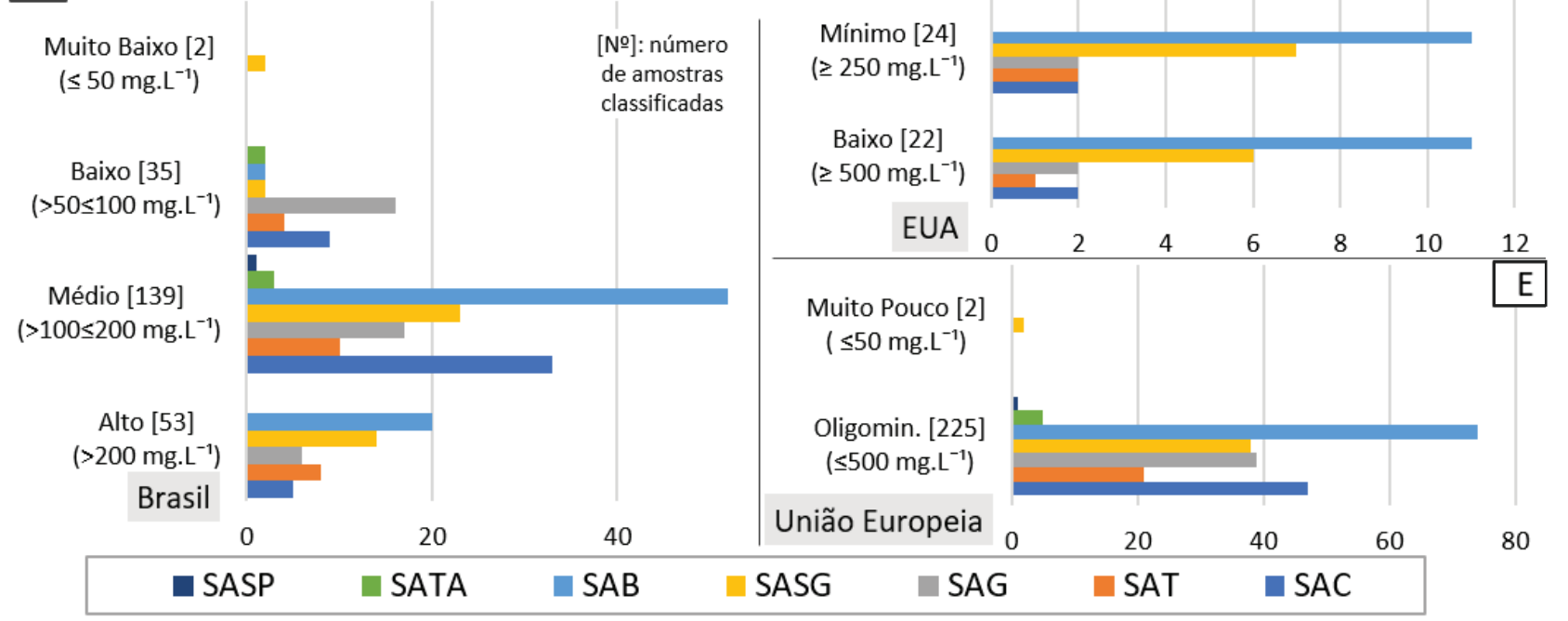

Figura 2. Concentrações dos STD nos aquíferos. A) Box-Plot com a distribuição empírica das concentrações; B) Análise estatística quanto ao STD; C) Classificação quanto o teor de sais, pela legislação brasileira (baseado em DNPM, 2015); D) Classificação mineral pelo STD na legislação dos Estados Unidos (baseado em U. S. Federal Government, 2019); E) Classificação mineral pelo STD na legislação da União Europeia (baseado em Parlamento Europeu, 2009).

Figure 2. Total Dissolved Solids (TDS - STD as Portuguese abbreviation) content in the aquifers. A) Box-Plot with empiric distribution of the concentrations; B) TDS Statistical Analysis of the aquifers; C) Classification based on solid content, by Brazilian legislation (based on DNPM, 2015); D) Mineral classification of TDS by The United States legislation (based on U. S. Federal Government, 2019); E) Mineral classification of TDS by the European Union legislation (based on Parlamento Europeu, 2009)

Dos demais elementos, o lítio é o que apresentou a segunda maior ocorrência nas águas subterrâneas (Fig. 4), sendo detectado em 41 poços (14,9\% do total), com concentrações variando entre 0,01 a 0,038 $\mathrm{mg} \mathrm{L}^{-1}$ de lítio. Quanto a distribuição espacial, mais da metade das amostras com concentrações de Lítio que permite a sua classificação, estão em poços que exploram águas subterrâneas do SAC (22 poços), seguido por amostras do SAT (sete poços), SAB (seis poços), SAG (três poços), SATA (dois poços) e SASG (um poço). No mapa da Fig. 4, nota-se a faixa de abrangência do elemento, bem distribuído sobre o SAC, enquanto no $S A B$, concentra-se no extremo oeste do estado.
Seguindo a ordem de ocorrência, o vanádio foi encontrado em amostras de 24 poços (8,7\% do total) (Fig. 4), com concentrações que variam de 0,0323 a 0,131 $\mathrm{mg} \mathrm{L}^{-1}$, (média de 0,067 $\mathrm{mg} \mathrm{L}^{-1} \pm$ 0,027). As águas denominadas como vanádicas estão distribuídas no Planalto Ocidental Paulista. A maior concentração de poços está associada aos basaltos do SASG, com 14 localidades, seguida do SAB com nove e do SAG com apenas duas amostras.

Para as águas classificadas como alcalinabicarbonatadas, com concentração mínima de $\mathrm{HCO}_{3}$ e $\mathrm{Na}$ (145 e $55 \mathrm{mg} \mathrm{L}^{-1}$ respectivamente), foram definidos quatro poços com potencial (1,45\% do total de pontos). As amostras atingiram 
Quadro 3. Comparativo das regulamentações sobre as águas minerais no Brasil, EUA, e Comunidade Europeia. [ ${ }^{(1)}$ DNPM (2015); (2) Brasil (1945); (3) Brasil (2014); (4) U.S. Federal Government (2019); (5) Parlamento Europeu (2009)]. Abreviação: STD: Sólidos Totais Dissolvidos; VMP. Valor máximo permitido.

Chart 3 Comparative of legislations about mineral water in Brazil, USA and European Community. [(1) DNPM (2015); (2) Brasil (1945); (3) Brasil (2014); (4) U.S. Federal Government (2019); (5) Parlamento Europeu (2009)]. Abbreviation: TDS (STD in Portuguese): Total Dissolved Solids, VMP. Maximum concentration allowed.

\begin{tabular}{|c|c|c|c|c|}
\hline \multicolumn{2}{|c|}{ Classificação } & Brasil & Estados Unidos ${ }^{(4)}$ & Comunidade Europeia ${ }^{(5)}$ \\
\hline \multirow{4}{*}{\multicolumn{2}{|c|}{$\begin{array}{l}\text { Pelo Teor de Sais } \\
\text { (STD) }\end{array}$}} & Muito Baixo: $\leq 50 \mathrm{ppm}^{(1)}$ & $\begin{array}{c}\text { Teor mínimo: } \geq 250 \\
\text { ppm }\end{array}$ & Muito Pouco: $\leq 50$ ppm \\
\hline & & Baixo: $>50 \leq 100$ ppm $^{(1)}$ & Baixo: $\leq 500$ ppm & $\begin{array}{c}\text { Pouco mineralizada: } \\
\leq 500 \mathrm{ppm}\end{array}$ \\
\hline & & Médio: $>100 \leq 200 \mathrm{ppm}^{(1)}$ & Alto: $\geq 1500 \mathrm{ppm}$ & Rica em sais: $\geq 1500 \mathrm{ppm}$ \\
\hline & & Alto: $>200 \mathrm{ppm}^{(1)}$ & & \\
\hline \multicolumn{2}{|c|}{ Bicarbonatada } & 200 ppm $\mathrm{NaHCO}_{3}^{(2)}$ & - & $600 \mathrm{HCO}_{3}$ \\
\hline \multicolumn{2}{|c|}{ Cálcica } & $480 \mathrm{ppm}$ de $\mathrm{Ca}$ em $\mathrm{Ca}\left(\mathrm{HCO}_{3}\right)_{2}{ }^{(2)}$ & - & $150 \mathrm{ppm} \mathrm{Ca}$ \\
\hline \multicolumn{2}{|c|}{ Magnesiana } & 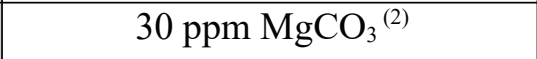 & - & $50 \mathrm{ppm} \mathrm{Mg}$ \\
\hline \multicolumn{2}{|c|}{ Ferruginosa } & $500 \mathrm{ppm} \mathrm{Fe}{ }^{(2)}$ & VMP 0,3 ppm Fe & $1 \mathrm{ppm} \mathrm{Fe}$ \\
\hline \multicolumn{2}{|c|}{ Sulfatada } & $100 \mathrm{ppm} \mathrm{SO}_{4}$ com $\mathrm{Na}, \mathrm{Mg}$ ou K$^{(2)}$ & $\begin{array}{c}\text { Contaminante } \geq 250 \\
\mathrm{ppm} \mathrm{SO}_{4}\end{array}$ & $200 \mathrm{ppm} \mathrm{SO}_{4}$ \\
\hline \multicolumn{2}{|c|}{ Cloretada } & $500 \mathrm{ppm} \mathrm{NaCl}^{(2)}$ & VMP $250 \mathrm{ppm} \mathrm{Cl}$ & $200 \mathrm{ppm} \mathrm{Cl}$ \\
\hline \multirow{4}{*}{ 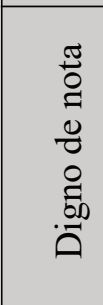 } & Fluoretada & $0,02 \mathrm{ppm} \mathrm{F}^{(3)}$ & - & $1 \mathrm{ppm} \mathrm{F}$ \\
\hline & Litinada & $0,01 \mathrm{ppm} \mathrm{Li}^{(3)}$ & - & - \\
\hline & Vanádica & $0,03 \mathrm{ppm} \mathrm{V}^{(3)}$ & - & - \\
\hline & Seleniada & $0,006 \mathrm{ppm} \mathrm{Se}^{(3)}$ & $\begin{array}{c}\text { Contaminante } \geq 0,05 \\
\text { ppm Se }\end{array}$ & - \\
\hline
\end{tabular}

concentrações entre 158 a 164 mg L-1de $\mathrm{HCO}_{3}$, todas de ocorrência na região central da Depressão Periférica (no SAT), e correspondem a 16\% dos poços do Sistema Aquífero Tubarão (Fig. 4).

As águas sulfatadas, definidas em função das concentrações de $\mathrm{SO}_{4}$ previsto pelo CAM, concomitantemente com os ânions $\mathrm{Na}, \mathrm{K}$, ou $\mathrm{Mg}$, foram reconhecidas em três poços, com concentrações média de $237 \mathrm{mg} \mathrm{L}^{-1}$, variando entre 138 e $287 \mathrm{mg} \mathrm{L}^{-1}$ de $\mathrm{SO}_{4}$. Essas amostras estão distribuídas na porção centro-leste do estado, em poços explorando águas do SAT e uma amostra ao norte, no Planalto Ocidental, captando águas no SASG (Fig. 4).

As concentrações de selênio permitem apenas o enquadramento de duas amostras, distribuídos em pontos opostos. As amostras contendo selênio ocorrem no extremo norte, no SAG com 0,019 $\mathrm{mg} \mathrm{L}^{-1}$, e no centro-sul, no SASG com 0,006 $\mathrm{mg} \mathrm{L}^{-1}$ (Fig. 4).

Uma análise da distribuição dos principais elementos químicos encontrados, permite definir um zoneamento hidromineral das águas nos aquíferos. O flúor ocorre por todo estado, com alguns locais com valores discrepantes, acima do máximo permitido para consumo. As águas minerais litinadas, têm ocorrência principal no SAC, e com concentrações relevantes nos demais aquíferos, com exceção do SASP. O vanádio é predominante no SASG, seguido do SAB e SAG. O sulfato ocorre em menor quantidade nos poços do SAT, com pontos também ricos em concentrações de alcalina bicarbonatadas. Por fim, o selênio de pouca expressão, ocorre no SAG e SASG. 


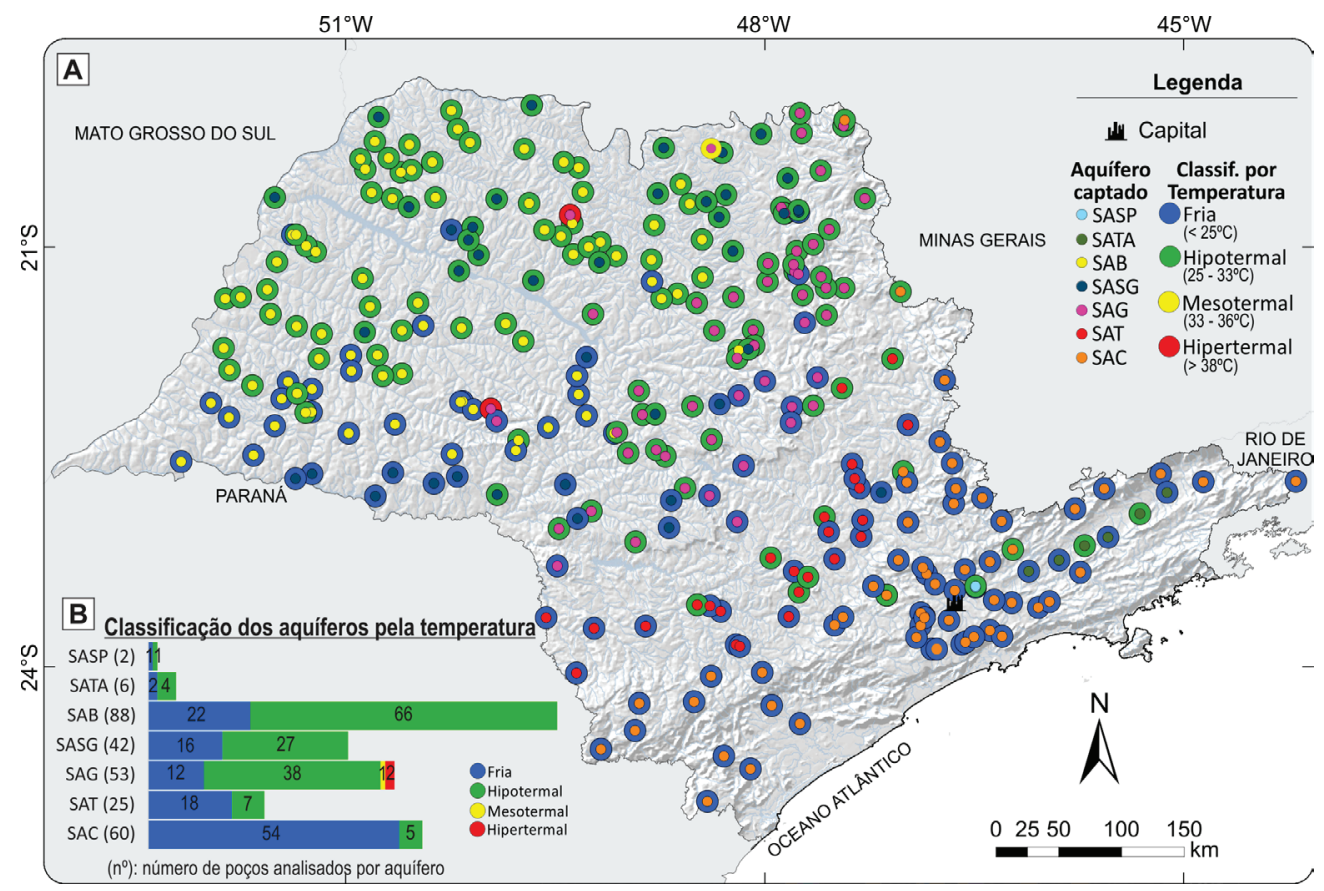

Figura 3. Classificação das amostras pela temperatura segundo CAM. A) Mapa de distribuição das temperaturas por amostra (os círculos maiores correspondem a classificação das águas pela temperatura, enquanto os menores são os aquíferos nas quais as amostras foram coletadas). B) O gráfico indica como essa classificação está distribuída por aquífero.

Figure 3. Sample's classification by temperature, second CAM. A) Samples' temperature distribution map (the bigger circles correspond to the temperature, while the small circles are the aquifers where the samples were collected from). $B$ ) The chart shows how this classification is distributed by aquifer.

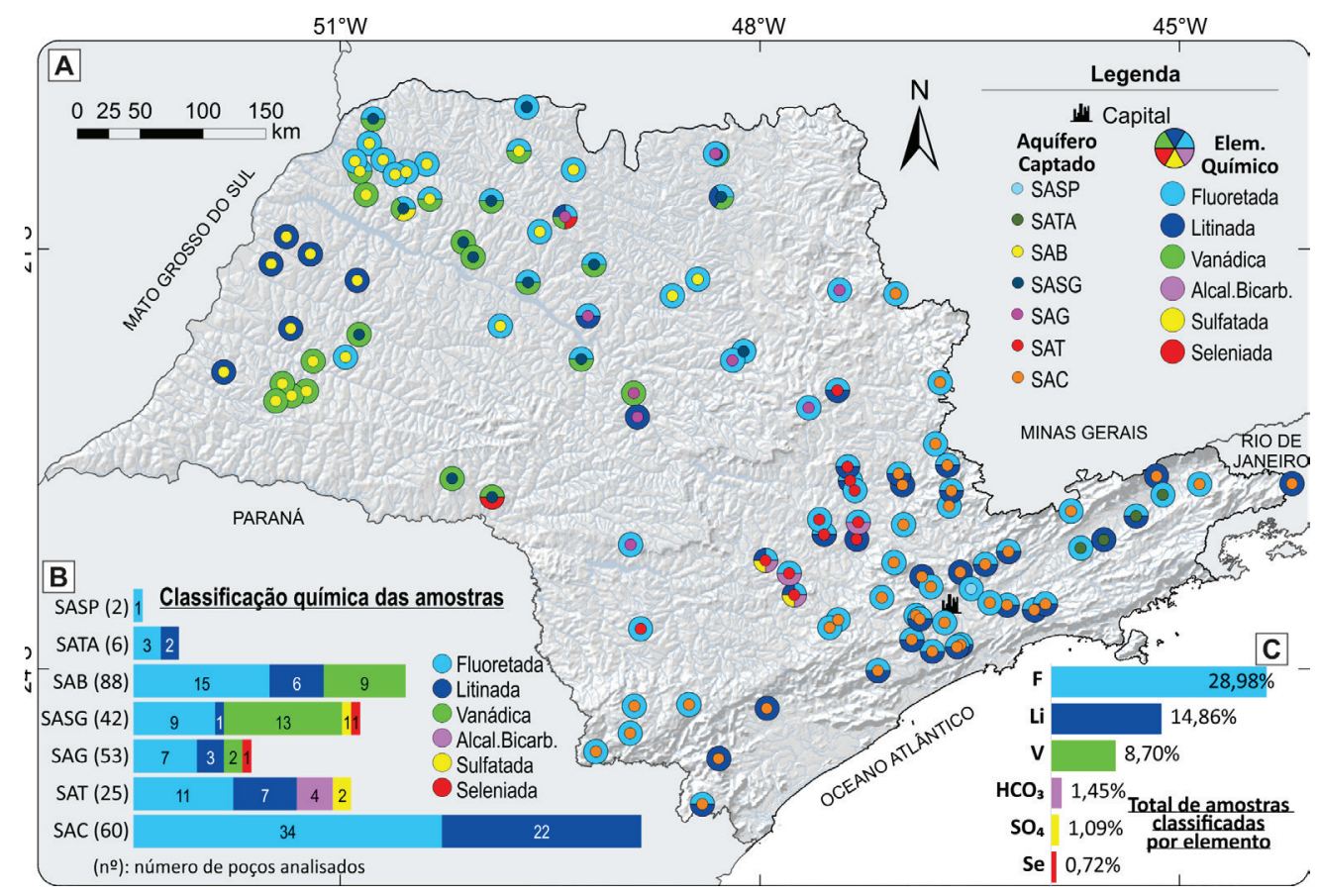

Figura 4. Classificação química das águas subterrâneas pelo CAM. A) Mapa de classificação química (os círculos maiores correspondem aos pontos com concentrações que atingiram o limite mínimo para classificação como água mineral, enquanto os círculos menores são os aquíferos em que as águas foram extraídas). B) Gráfico de classificação por aquífero. C) Porcentagem do total de amostras classificadas pela composição química. Abreviações F: Flúor; Li: Lítio; V: Vanádio; $\mathrm{SO}_{4}$ : Sulfato; $\mathrm{HCO}_{3}$ : Bicarbonato; Se: Selênio.

Figure 4. Chemical Classification of the groundwaters, by CAM. A) Chemical classification map (the bigger circles represent the wells which has the minimum of concentration to be classified as mineral water, while the small circles are the aquifers where those samples were collected from).B) Classification chart per aquifer. C) Percentage of the total

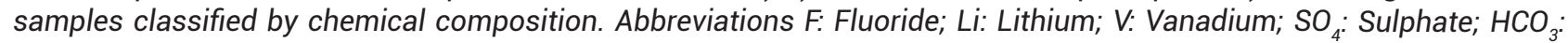
Bicarbonate; Se: Selenium. 
O quimismo das águas subterrâneas é função do arcabouço geológico (litologia) e o tempo de interação água-rocha. A variação dos elementos químicos apresentada no presente trabalho, permite admitir que aquíferos formados por distintas litologias apresentam águas subterrâneas de composições químicas caracterizadas por diferentes íons dissolvidos. Desse modo, é possível delimitar zonas de interesse hidromineral ao longo do estado de São Paulo.

\section{Conclusão e considerações finais}

A base de dados da Companhia Ambiental do estado de São Paulo, de domínio público, analisada no presente estudo, nunca fora utilizada com a finalidade exploratória, e possibilitou a definição de um zoneamento hidrotermal, caracterizando ao longo do estado de São Paulo, áreas com diferentes potenciais hidrominerais, segundo as diretrizes brasileiras de classificação das águas minerais, tanto em relação à temperatura quanto em relação à concentração de elementos químicos (oligoelementos).

Quanto à temperatura, nota-se um incremento sentido sudeste para noroeste do estado. Esse aumento reflete áreas de captação em maiores profundidades. Em regiões de águas mais frias, como no caso dos pontos do SAC, as profundidades médias dos poços não ultrapassam 200 metros e, contrastam com as águas mais quentes que ocorrem no SAG, relacionadas à poços profundos.

As águas dos aquíferos paulistas são, predominantemente, caracterizadas como pouco mineralizadas. As maiores concentrações de sais, todavia, pertencem a alguns poços do SASG, SAT e SAB, e refletem, na maioria dos casos, em uma classificação química mista, segundo o CAM. Nesse contexto, a classificação química pelos elementos dispostos pela legislação brasileira, todos os aquíferos têm potencial para classificação como mineral, através de elementos como selênio, sulfato, flúor, lítio, vanádio e bicarbonato. A principal ocorrência foi de águas minerais fluoretadas, seguidas em ordem decrescente pelas águas litinadas, vanádicas, alcalina-bicarbonatadas, sulfatadas e seleniadas.

Desse modo, constata-se um zoneamento hidromineral das águas. As águas com lítio concentram-se no sudeste do estado, com maior ocorrência no SAC, seguido do SAT, além do SAB no extremo noroeste. As águas vanádicas ocorrem preferencialmente no Planalto Ocidental Paulista, nos poços dos SASG e em zonas mais rasas no SAB. As águas alcalinas bicarbonatadas ocorrem na região central do SAT. As águas sulfatadas são classificadas como mistas, com ocorrência conjunta com as litinadas e alcalina bicarbonatada no SAT e, em conjunto com o vanádio no SASG. O selênio ocorre de modo aleatório, o que impossibilita determinar seu zoneamento mineral.

É sabido que no período de publicação do CAM, em 1945, o enfoque das classificações das águas minerais era baseado, principalmente, nas instâncias minero e termomedicinais. Nesse sentido, a temperatura e radioatividade foram tão importantes na caracterização dessas águas, tanto quanto a composição química. O que ocorre, hoje em dia, é que as águas potáveis de mesa, podem ser denominadas como minerais, através da temperatura na fonte, embora não apresentem concentrações mínimas de minerais para tal. Como atualmente o ramo da produção das águas minerais é destinado ao envase, não haveria mais a necessidade dessa classificação, uma vez que o consumidor não seria beneficiado por tais efeitos. Assim, faz-se necessário evoluir o pensamento, restringindo a classificação como mineral, somente quando destinada a balneários.

A rotulação das águas pelos elementos dignos de nota, foi preponderante nesse trabalho. Embora pela legislação atual, o CAM, não conste os limites mínimos, a Portaria n 540/2014 determina tais limites e os caracterizam como "elementos químicos raros". Todavia, o teor mínimo de 0,02 $\mathrm{mg} \mathrm{L}^{-1}$, permite a classificação de todos os aquíferos, o que contradiz com o termo "raro". Fica evidente que, a diretriz brasileira para classificação das águas com o flúor é demasiadamente permissiva, fato que não ocorreria na lei europeia, que estabelece valor mínimo de 1 mg L-1. Assim, somente 2,90\% das águas amostradas poderiam ser rotuladas como água mineral fluoretada. 
Agradecimentos. Os autores gostariam de agradecer ao Prof. Dr. Paulo Alves de Souza, editor chefe e a Profa. Dra. Tais Freitas, membros da equipe editorial da revista Pesquisa em Geociências da UFRGS, e ao revisor anônimo pelos comentários feitos no manuscrito original, que em muito contribuíram para a sua melhoria e a FAPESP, pela concessão de recursos que possibilitaram a realização da pesquisa (Processo 2018/06666-4).

\section{Referências}

Barison, M.R. \& Chang, H.K. 2002. Caracterização hidroquímica da porção meridional do Aquífero Bauru no Estado de São Paulo. In: Congresso Brasileiro de Águas Subterrâneas, 12, 2002. Cidade, Anais... Cuiabá, ABAS, p. 1-12.

Bertolo, R., Hirata, R. \& Fernandes, A. 2007. Hidrogeoquímica das águas minerais envasadas do Brasil. Revista Brasileira de Geociências, 37(3): 515-529.

Brasil. 1945. Decreto-Lei no 7.841, de 8 de agosto de 1945: Código de Águas Minerais. Brasília, Brasil.

Brasil. 2014. Ministério de Minas e Energia. Departamento Nacional de Produção Mineral - DNPM. Portaria n 540, de 18 de dezembro de 2014.

Bulia, I.L. \& Enzweiler, J. 2018. The hydrogeochemistry of bottled mineral water in São Paulo state, Brazil. Journal of Geochemical Exploration, 188: 43-54.

Campos, H.C.N.S. 1993. Caracterização e Cartografia das Províncias Hidrogeoquímicas do Estado de São Paulo. São Paulo. 117p. Tese de Doutorado, Programa de Pós-graduação em Geoquímica e Geotetônica, Instituto de Geociências, Universidade de São Paulo.

CERH. Conselho Estadual de Recursos Hídricos. 1999. Relatório de situação dos recursos hídricos do Estado de São Paulo. São Paulo:Comitê Coordenador do Plano Estadual de Recursos Hídricos, 128p.

CETESB. Companhia Ambiental do Estado de São Paulo. 1998. Relatório de Qualidade das Águas Subterrâneas do Estado de São Paulo 1997. São Paulo.

CETESB. Companhia Ambiental do Estado de São
Paulo. 2010. Relatório de qualidade das águas subterrâneas no Estado de São Paulo: 20072009 (recurso eletrônico) / equipe técnica Modesto et al. São Paulo, Série Relatórios/ CETESB, 258p.

CETESB. Companhia Ambiental do Estado de São Paulo. 2016. Qualidade Das Águas Subterrâneas No Estado De São Paulo 20132015. São Paulo, Série Relatórios/CETESB, $308 p$.

Chang, H.K., Stradioto, M.R. \& Silva, F.P. e. 2016. Tipos hidroquímicos do Sistema Aquífero Bauru no Estado de São Paulo. Águas Subterrâneas, 30(2): 224-245.

DAEE/IG/IPT/CPRM. Departamento de Água e Energia Elétrica, Instituto Geológico, Instituto de Pesquisas Tecnológicas \& Serviço Geológico do Brasil. 2005. Mapa de Águas Subterrâneas do Estado de São Paulo, Escala 1:7.000.000: Nota Explicativa / Rocha, G. (Coord.). São Paulo: DAEE/IG/IPT/CPRM, $119 p$.

DAEE/UNESP. Departamento de Águas e Energia Elétrica, Universidade Estadual Paulista. 1980. Mapa geológico do Estado de São Paulo. Escala 1: 250.000. São Paulo: Convênio DAEE/UNESP/Secretaria de Obras e do Meio Ambiente do Estado de São Paulo. (Compilação eletrônica Lebac/ IGCE/ Unesp, 2009).

DNPM. Departamento Nacional de Produção Mineral. 2015. Estudo diagnóstico das Águas minerais e potáveis de mesa do Brasil. Coord. Geral: Queiroz, E.T e Pontes C.H.C. Brasília, DNPM, 474p.

DNPM. Departamento Nacional de Produção Mineral. 2017. Sumário Brasileiro Mineral 2017: Água Mineral / Assirati, D.M. São Paulo, DNPM-SP, 2p

Ezaki, S., Oda, G.H., Iritani, M.A., Veiga, C. \& Stradioto, M.R. 2014. Hidroquímica dos aquíferos Tubarão e Cristalino na região de Indaiatuba-Rafard, Estado de São Paulo. Pesquisas em Geociências, 41(1): 65-79.

Gastmans, D., Reis, M.M. \& Chang, H.K. 2012. Geotermometria das águas hipertermais do Sistema Aquífero Guarani no estado de São Paulo. Revista Brasileira de Geociências, 42(Suppl 1): 208-225.

IBGE. Instituto Brasileiro de Geografia e 
Estatística. 2017. Dados estatísticos Panorama do Estado de São Paulo. Disponível em: https://cidades.ibge.gov.br/brasil/sp/ panorama. Acessado em: 15 maio 2018.

Iritani, M.A. \& Ezaki, S. 2009. As águas subterrâneas do Estado de São Paulo. $2^{\mathrm{a}}$ ed. ed. São Paulo: SMA/IG, 104p.

LAMIN. Rede de Laboratórios de Análises Minerais, Serviço Geológico Brasileiro, CPRM. 2015. Estudo Comparativo entre as Classificações Brasileira, Norte-Americana e da Comunidade Europeia. Disponível em: <http://www.cprm.gov.br/publique/RedesInstitucionais/Rede-de-Laboratorios-deAnalises-Minerais---Rede-LAMIN/EstudoComparativo-entre-as-ClassificacoesBrasileira\%2C-Norte-Americana-e-daComunidade-Europeia-3526.html>. Acesso em: 10 fev. 2020.

Parlamento Europeu. 2009. Directiva 2009/54/ CE do Parlamento Europeu e do Conselho de 18 de junho de 2009 relativa à exploração e à comescialização de águas minerais naturais.

Porowski A. 2018. Mineral and Thermal Waters. In: Meyers RA (Ed.). Encyclopedia of Quaternary Science. $2^{\text {nd }}$ ed. Springer Science Businnes Media LLC 2018, p. 149-181.

Porowski, A., Rman, N., Fórizs, I. \& Lamoreaux, J. 2019. Introductory Editorial Thematic Issue: "Mineral and thermal waters". Environmental Earth Sciences, Springer Berlin Heidelberg, 78(16): 526-527.

Quaggio, C.S., Gastmans, D., Kirchheim, R. \& Batista, L.V. 2018. Variações na composição das águas subterrâneas do Sistema Aquífero Serra Geral em território brasileiro e sua relação com anomalias hidrogeoquímicas. Águas Subterrâneas, 32(3): 283-294.

Rodwann Jr., J.G. 2018. Bottled Water: 2017 Staying Strong. Disponível em: https://www. bottledwater.org/public/BMC2017_BWR_ StatsArticle.pdf. Acessado em: 10 set. 2019.
Rolim, G.S., Camargo, M.B.P. de, Lania, D.G. \& Moraes, J.F.L. de. 2007. Classificação climática de Köppen e de Thornthwaite e sua aplicabilidade na determinação de zonas agroclimáticas para o estado de são Paulo. Bragantia, Instituto Agronômico, 66(4): $711-$ 720.

Roque, D.A., Gastmans, D., Quaggio, C.S. \& Santarosa, L.V. 2018. Hidroquímica do Aquífero Pré-Cambriano no estado de São Paulo Resultados Preliminares. In: CONGRESSO BRASILEIRO DE ÁGUAS SUBTERRÂNEAS, 20. 2018, Campinas, Anais... Campinas, ABAS, p. $1-4$

Selinus, O. 2005. Essentials of Medical Geology: Impacts of the Natural Environment on Public Health. Burlinton, Elsevier Academic Press, $812 p$.

Shvartsev, S.L. 2008. Geochemistry of fresh groundwater in the main landscape zones of the Earth. Geochemistry International, Pleades Publishing, 46(13): 1285-1398.

Szikszay, M. \& Teissedre, J.M. 1981. "Águas Minerais" no estado de São Paulo. Boletim IG, Instituto de Geociências, USP, 12: 11-22.

U.S. Federal Government. 2019. 21 CFR 165: eCFR - Code of Federal Regulations - Beverages, data is current as of September 19, 2019.

Vidal, A.C. \& Chang, H.K. 2002. Caracterização Hidroquímica dos Aquíferos da Bacia de Taubaté. Revista Brasileira de Geociências, 32(2): $267-276$. 